VOL. $5(1971), 75-86$.

\title{
Divisibility of binary relations
}

\author{
D.G. Fitz-Gerald and G.B. Preston
}

\begin{abstract}
In his paper in Mat. Sb. (N.S.) 61 (103) (1963), ZareckiT associated with any binary relation $\alpha$ an ordered pair, $\left(L_{\alpha}, M_{\alpha}\right)$, say, of lattices and showed that $\alpha$ is a left [right] divisor of $\beta$ if and only if $L_{\alpha} \supseteq L_{\beta}\left[M_{\alpha} \supseteqq M_{\beta}\right]$. We provide an alternative proof of this result by embedding the category of relations in the category of sets. Our approach provides a unified treatment of several hitherto independent results, and gives new results for the category of partial transformations.
\end{abstract}

\section{Preliminaries}

We shall be dealing with four categories, the category of relations and three of its subcategories. We regard a category as consisting of objects and maps. In the category of relations the class of objects is the class of all sets and the class of maps is the class of all ordered triples $(X, \alpha, Y)$, where $X$ and $Y$ are sets and where $\alpha \subseteq X \times Y$. The product $(X, \alpha, Y)(U, \beta, V)$ is defined if and only if $Y=U$ and, when defined, it equals $(X, \alpha \circ \beta, V)$. Here $\alpha \circ \beta$ denotes the composition of $\alpha$ and B :

$$
\alpha \circ \beta=\{(x, v) \mid(x, y) \in \alpha \text { and }(y, v) \in \beta \text {, for some } y\} \text {. }
$$

We shall frequently denote $(X, \alpha, Y)$ by $\alpha$ in what follows and consequently write the product $(X, \alpha, Y)(U, \beta, V)$ simply as $\alpha \beta$. We shall denote the category of relations by $\underline{\underline{R}}$.

Received 16 January 1971. This work was completed while the first author held a Monash Graduate Scholarship, and while the second author was a visitor to the Department of Mathematics, University of Stirling, to whom he wishes to record his gratitude for a stimulating year. 
The following notations will be convenient. Let $(X, \alpha, Y)$ be a map of $\underline{\mathrm{R}}$. Then we set

$$
\begin{aligned}
& \Delta(\alpha)=\{x \in X \mid(x, y) \in \alpha \text { for some } y\}, \\
& \nabla(\alpha)=\{y \in Y \mid(x, y) \in \alpha \text { for some } x\} .
\end{aligned}
$$

The category of sets, consisting of those maps $(X, \alpha, Y)$ of $\underline{\underline{R}}$ for which $\alpha: X \rightarrow Y$, we shall denote by $\underline{\underline{S}}$. The category of partial transformations, consisting of those maps $(X, \alpha, Y)$ of $\underline{\underline{R}}$ for which $\alpha: \Delta(\alpha) \rightarrow y$, we shall denote by $\underline{\underline{P}}$. Finally, we shall denote by $\underline{I}$ the category of one-to-one partial transformations, consisting of those maps $(X, \alpha, Y)$ of $\underline{\underline{P}}$ for which $\alpha$ is a one-to-one mapping of $\Delta(\alpha)$ onto $\nabla(\alpha)$. I is then a subcategory of $\underline{\underline{P}}$. Each of the categories $\underline{\underline{R}}, \underline{\underline{S}}, \underline{\underline{P}}$ and $\underline{\underline{I}}$ has the same class of objects.

If $\alpha$ and $\beta$ are maps of a category $\underline{\underline{K}}$ then we shall write $\alpha \lambda_{\underline{\underline{K}}} \beta\left[\alpha \rho_{\underline{\underline{K}}} \beta\right]$, to mean that there exists a map $\gamma$ in $\underline{\underline{K}}$ such that $\alpha=\gamma \beta[\alpha=\beta \gamma]$. We write $\alpha \underline{\underline{K}} \beta\left[\alpha R_{\underline{K}} \beta\right]$ to mean that

$$
\alpha \lambda_{\underline{\underline{K}}}^{\beta} \text { and } \beta \lambda_{\underline{\underline{K}}}^{\alpha}\left[\alpha \underline{\underline{K}}_{\underline{\underline{K}}}^{\beta} \text { and } \beta \underline{\underline{\underline{K}}}^{\alpha}\right]
$$

both hold.

We shall say that an element $\alpha$ of a category $\underline{\underline{K}}$ is regular, if there is a $\beta$ in $\underline{\underline{K}}$ such that $\alpha \beta \alpha=\alpha$; there then exists $\gamma$ in $\underline{\underline{K}}$, for example take $\gamma=\beta \alpha \beta$, such that $\alpha \gamma \alpha=\alpha$ and $\gamma \alpha \gamma=\gamma ; \gamma$ will be called an inverse of $\alpha . \underline{\underline{K}}$ is said to be regular if each of its maps is regular. For example, $\underline{\underline{S}}$ is a regular category and $\underline{\underline{R}}$ is not regular.

\section{Divisibility in $\underline{I}, \underline{P}$ and $\underline{S}$}

The set of all maps $(X, \alpha, X)$, for a fixed set $X$, in each of the categories $\underline{\underline{I}}, \underline{\underline{P}}$ and $\underline{\underline{\mathrm{S}}}$ forms a semigroup; these semigroups are $I_{X}$, the symmetric inverse semigroup on $X, P_{X}$, the semigroup of partial transformations of $X$, and $T_{X}$, the semigroup of transformations of $X$, respectively.

In the following theorem we show that certain well-known characterizations of left and right divisibility of elements in $T_{X}$ 
(Preston [2]), anä $I_{X}$ (Reilly [3]) extend in a natural fashion to $\underline{\underline{S}}$, and $I$ respectively.

If $\alpha \subseteq X \times Y$ then we write

$$
\alpha^{-1}=\{(y, x) \mid(x, y) \in \alpha\} ;
$$

for $A \subseteq X$ we write

$$
A \alpha_{*}=\{y \in Y \mid(a, y) \in \alpha \text {, for some } a \text { in } A\} \text {; }
$$

and for $B \subseteq Y$ we write

$$
B \alpha^{*}=B\left(\alpha^{-1}\right)_{*}
$$

Moreover, if $(X, \alpha, Y)$ is a map in $\underline{\underline{P}}$, so that $\alpha \circ \alpha^{-1}$ is a partial equivalence relation on $X$, that is an equivalence relation on $\Delta(\alpha)$, then we denote by $X /\left(\alpha \circ \alpha^{-1}\right)$ the set of $\alpha \circ \alpha^{-1}$-equivalence classes; so that $X /\left(\alpha \circ \alpha^{-1}\right)=\Delta(\alpha) /\left(\alpha \circ \alpha^{-1}\right)$. If $\alpha$ is empty, so that $\Delta(\alpha)$ is then empty, we take $X /\left(\alpha \circ \alpha^{-1}\right)$ to mean the empty set.

For any set $X$ we shall denote by ${ }^{2} X$ the set ${ }^{l_{X}}=\{(x, x) \mid x \in X\}$.

THEOREM 1. Let $\underline{\underline{K}}$ be one of the categories $\underline{\underline{I}} \underline{\underline{\mathrm{P}}}$ and $\underline{\underline{\mathrm{S}}}$. Let $(X, \alpha, Y)$ and $(U, \beta, V)$ be mops in $\mathrm{K}$. Then

(i) $\alpha \lambda_{\underline{K}} \beta$ if and only if $Y=V$ and $\nabla(\alpha) \subseteq \nabla(\beta)$; and

(ii) $\alpha \rho_{\underline{\underline{K}}} \beta$ if and only if $x=U, \Delta(\alpha) \subseteq \Delta(\beta)$, and setting $D=\Delta(\beta) \backslash \Delta(\alpha)$,

$$
(D \times D) \cup\left(\alpha \circ \alpha^{-1}\right) \supseteqq \beta \circ \beta^{-1} .
$$

Proof. We prove the result for the category $\underline{\underline{P}}$; for the other two categories we may proceed similarly or may, as follows from the next lema, deduce the result from that for $P$.

(i) If $\alpha \lambda_{\underline{\underline{P}}} \beta$ then there exists $\gamma$ in $\underline{\underline{P}}$ such that $\alpha=\gamma \beta$, that is there exists $(W, \gamma, z)$, say, such that $(X, \alpha, Y)=(W, \gamma, Z)(U, \beta, V)$. Then $X=W, Z=U, Y=V$, and 
$\alpha=\gamma \circ \beta$. From these equations we deduce that

$$
\nabla(\alpha)=X \alpha_{*}=\left(X Y_{*}\right) \beta_{*} \subseteq U \beta_{*}=\nabla(\beta) \text {. }
$$

Conversely, suppose that $Y=V$ and that $\nabla(\alpha) \subseteq \nabla(\beta)$. Define $\gamma: \Delta(\alpha) \rightarrow U$ by choosing, for each $x$ in $\Delta(\alpha)$, xy to be any element in $U$ such that $(x \gamma) \beta=x \alpha$; this is possible because $\nabla(\alpha) \subseteq \nabla(\beta)$. Then $(X, \gamma, U)$ is a map of $\underline{\underline{P}}$ and $(X, \alpha, Y)=(X, \gamma, U)(U, B, V)$; which completes the proof.

(ii) If $\alpha \rho_{\underline{\underline{p}}} \beta$, then there exists $\delta$ in $\underline{\underline{P}}$ such that $\alpha=\beta \delta$, that is there exists $(W, \delta, Z)$, say, such that $(X, \alpha, y)=(U, \beta, V)(W, \delta, Z)$. Then $X=U, V=W, Y=Z$ and $\alpha=\beta \circ \delta$. From these we have $\Delta(\alpha)=\nabla\left(\alpha^{-1}\right)=\nabla\left(\delta^{-1} \circ \beta^{-1}\right)=\left(Y\left(\delta^{-1}\right)_{*}\right)\left(\beta^{-1}\right)_{*} \subseteq v\left(\beta^{-1}\right)_{*}=\nabla\left(\beta^{-1}\right)=\Delta(\beta)$. Further, if $\left(x, x^{\prime}\right) \in \beta \circ \beta^{-1}$ and $x \in \Delta(\alpha)$, then there exists $y$ such that $(x, y) \in \alpha=\beta \circ \delta$; and so there exists $v$ such that $(x, v) \in \beta$ and $(v, y) \in \delta$. Since $\beta$ is a mapping, $\left(x, x^{\prime}\right) \in \beta \circ \beta^{-1}$ implies that $\left(x^{\prime}, v\right) \in \beta$. Hence $\left(x^{\prime}, y\right) \in \beta \circ \delta=\alpha$; hence $\left(x, x^{\prime}\right) \in \alpha \circ \alpha^{-1}$. Thus $\beta \circ \beta^{-1} \cong\left(\alpha \circ \alpha^{-1}\right) \cup(D \times D)$.

Conversely, suppose that $X=U, \Delta(\alpha) \subseteq \Delta(\beta)$, and that $(D \times D) \cup\left(\alpha \circ \alpha^{-1}\right) \supseteq \beta \circ \beta^{-1}$. Since $\Delta(\alpha) \subseteq \Delta(\beta),(D \times D) \cup\left(\alpha \circ \alpha^{-1}\right)$ and $\beta \circ \beta^{-1}$ are each equivalences on $\Delta(\beta)$. Thus each $\alpha \circ \alpha^{-1}$-class is a union of $\beta \circ \beta^{-1}$-classes. Set $E=(\Delta(\alpha)) \beta_{*}$ and define $\delta: E \rightarrow Y$, thus: for $e \in E$, choose $x$ in $\Delta(\alpha)$ so that $x \beta=e$; then define $e \delta$ to be $x \alpha$. If also $x^{\prime} \beta=e$, then $\left(x, x^{\prime}\right) \in \beta \circ B^{-1} \subseteq\left(\alpha \circ \alpha^{-1}\right) \cup(D \times D)$ and, since $x \in \Delta(\alpha)$, therefore $\left(x, x^{\prime}\right) \in \alpha \circ \alpha^{-1}$. Thus $x \alpha=x^{\prime} \alpha ;$ consequently $\delta$ is well-defined. Hence we have

$$
(X, \alpha, Y)=(U, \beta, V)(V, \delta, Y) ;
$$

which completes the proof.

REMARK I. The condition 


$$
(D \times D) \cup\left(\alpha \circ \alpha^{-1}\right) \supseteq \beta \circ \beta^{-1}
$$

could be replaced by the equivalent but more symmetric inequality

$$
(X \backslash \Delta(\alpha)) \times(X \backslash \Delta(\alpha)) \cup\left(\alpha \circ \alpha^{-1}\right) \supseteqq(X \backslash \Delta(\beta)) \times(X \backslash \Delta(\beta)) \cup\left(\beta \circ \beta^{-1}\right) .
$$

REMARK 2. In the categories $\underline{\underline{I}}$ and $\underline{\underline{S}}$ result ( $i i)$ may be simplified. If $\alpha$ is a map in $\underline{\underline{I}}$ then $\alpha \circ \alpha^{-1}={ }^{2} \Delta(\alpha)$, and similarly for $\beta$. Hence the final condition in $(i i)$ becomes superfluous and we have:

$$
\alpha \rho_{\underline{I}} \beta \quad \text { if and only if } X=U \text { and } \Delta(\alpha) \subseteq \Delta(\beta) \text {. }
$$

This is the result of Reilly already referred to [3].

If $\alpha$ and $\beta$ are maps in $\underline{\underline{S}}$ and $X=U$ then necessarily $\Delta(\alpha)=\Delta(\beta)=X$ and hence $D$ is empty. Thus we have

$$
\alpha \rho_{\underline{S}} \beta \text { if and only if } X=U \text { and } \alpha \circ \alpha^{-1} \supseteq \beta \circ \beta^{-1} \text {. }
$$

This is the result of Preston already referred to [2].

COROLLARY. Let $\underline{\underline{K}}$ be one of the categories $\underline{\underline{\mathrm{I}}} \underline{\underline{\mathrm{P}}}$ and $\underline{\underline{\mathrm{S}}}$. Let $(X, \alpha, Y)$ and $(U, \beta, V)$ be mops in $\underline{\underline{K}}$. Then

(i) $\alpha \underline{\underline{K}}^{\beta}$ if and only if $y=V$ and $\nabla(\alpha)=\nabla(\beta)$; and

(ii) $\alpha \mathrm{R}_{\underline{\underline{K}}}^{\beta}$ if and only if $X=U$ and $\alpha \circ \alpha^{-1}=\beta \circ \beta^{-1}$.

REMARK. For the category I, $\alpha \circ \alpha^{-1}=\beta \circ \beta^{-1}$ if and only if $\Delta(\alpha)=\Delta(\beta)$. Hence $\alpha R_{\underline{I}}^{\beta}$ if and only if $X=U$ and $\Delta(\alpha)=\Delta(\beta)$.

We now prove the lemma, mentioned in the proof of Theorem 1, which enables the results of Theorem $I$, for $\underline{\underline{S}}$ and for $\underline{\underline{I}}$, to be deduced from the result for $\underline{\underline{P}}$.

LEMMA 1. Let $\underline{\underline{L}}$ be a regular subcategory of the category $\underline{\underline{\mathrm{K}}}$. Let $\alpha, \beta$ be maps in $\underline{\underline{L}}$. Then $\alpha \lambda_{\underline{\underline{L}}} \beta\left[\alpha \rho_{\underline{\underline{L}}} \beta\right]$ if and only if $\alpha \lambda_{\underline{\underline{K}}} \beta\left[\alpha \rho_{\underline{\underline{K}}} \beta\right]$.

Proof. Clearly $\alpha \lambda_{\underline{\underline{L}}} \beta$ implies $\alpha \lambda_{\underline{\underline{K}}}{ }^{\beta}$. Suppose, conversely, that $\alpha, \beta$ are in $\underline{L}$ and that $\alpha \lambda_{K^{\beta}} \beta$. Thus there exists $\gamma$, say, in $\underline{\underline{K}}$ such that $\alpha=\gamma \beta$. Let $B^{\prime}$ be an inverse of $\beta$ in $\underline{\underline{I}}$. Then $\gamma \beta=\gamma B B^{\prime} \beta$, 
and $\gamma \beta=\alpha \in \underline{L}$ and $\beta^{\prime} \in \underline{\underline{L}}$, so that $\gamma \beta \beta^{\prime} \in \underline{\underline{L}} ;$ and hence $\alpha \lambda_{\underline{\underline{L}}} \beta$.

The bracketed assertion follows dually.

Since $\underline{S}$ and $\underline{I}$ are regular subcategories of $\stackrel{P}{\underline{P}}$ we may apply the lemma, as already claimed, to deduce immediately from the result for $\underline{\underline{P}}$, the results of Theorem $I$ for $\underline{\underline{S}}$ and for $\underline{\underline{I}}$.

Before turning to $\underline{\underline{R}}$ we show that we can subsume another situation under our treatment.

If we take any regular subcategory of $\underline{\underline{I}}$ then, from the lemma, it follows that the characterizations of divisibility that have been obtained for I serve to characterize divisibility in the subcategory. In particular these characterizations apply to any inverse subsemigroup of a symmetric inverse semigroup $I_{X}$. Thus we have the next theorem (Munn [1]).

THEOREM 2. Let $S$ be an inverse semigroup of one-to-one partial transformations of a set $X$. Let $\alpha, \beta \in S$. Then $\alpha \lambda \beta[\alpha \rho \beta, \alpha L \beta, \alpha R \beta]$ in $S$ if and only if $\nabla(\alpha) \subseteq \nabla(\beta)[\Delta(\alpha) \subseteq \Delta(\beta), \nabla(\alpha)=\nabla(\beta)$, $\Delta(\alpha)=\Delta(\beta)]$.

If we apply Lemma $I$ to any inverse semigroup $S$, making use of the faithful representation of $S$ as an inverse subsemigroup of $I_{S}$, then we quickly find that, for $\alpha, \beta$ in $S, \alpha \lambda \beta[\alpha \rho \beta, \alpha L \beta, \alpha R \beta]$ in $S$ if and only if

$$
\alpha^{-1} \alpha \leq \beta^{-1} \beta\left[\alpha \alpha^{-1} \leq \beta \beta^{-1}, \alpha^{-1} \alpha=\beta^{-1} \beta, \alpha \alpha^{-1}=\beta \beta^{-1}\right] \text {. }
$$

\section{3. $\underline{R}$ regarded as a subcategory of $\underline{S}$}

We denote by $P(A)$ the set of all subsets of a set $A$.

We extend the mappings $\alpha \leftrightarrow \alpha_{*}$ and $\alpha \leftrightarrow \alpha^{*}$, introduced in the second section, and, for $(X, \alpha, Y)$ a map in $\underline{\underline{R}}$, we define $(X, \alpha, Y) \rightarrow(X, \alpha, Y)_{*}=\left(P(X), \alpha_{*}, P(Y)\right)$ and $(X, \alpha, Y) \leftrightarrow(X, \alpha, Y)^{*}=\left(P(Y), \alpha^{*}, P(X)\right)$. We again, where convenient, denote $(X, \alpha, Y)$ by $\alpha$; so that now $\alpha_{*}$ and $\alpha^{*}$ each has two meanings, the relevant one being decided by the context. 
It will be convenient also to extend the meaning of $\alpha^{-1}$. We define for a map $(X, \alpha, Y)$ in $\underline{\underline{R}}(X, \alpha, Y)^{-1}$ to be $\left(y, \alpha^{-1}, X\right)$. Again there will be no ambiguity in denoting $(X, \alpha, Y)$ simply by $\alpha$ and hence $(X, \alpha, Y)^{-1}$ by $\alpha^{-1}$, when convenient. For $\alpha$ a map in $\underline{\mathrm{R}}$ we then have, with the extended meaning also, that $\alpha^{*}=\left(\alpha^{-1}\right)_{*}$.

The next result is easily verified.

\section{LEMMA 2.}

(i) $\alpha \mapsto \alpha_{*}, \alpha$ a map in $\underline{\underline{R}}$, is a co-variant functor embedding $\underline{\underline{R}}$ in $\underline{\underline{S}}$.

(ii) $\alpha \mapsto \alpha^{*}, \alpha$ a map in $\underline{\underline{R}}$, is a controvariant functor embedding $\stackrel{\mathrm{R}}{=}$ in $\stackrel{\mathrm{S}}{=}$.

We now use this embedding to show that left and right divisibility in $\underline{\underline{R}}$ can be characterized by the conditions we already have for $\underline{\underline{S}}$. Since $\underline{P}$ is a regular subcategory of $\underline{\underline{R}}$, because of Lemma 1 , the same embedding will enable us to deduce the characterizations of divisibility in $\underline{\underline{\underline{p}}}$, of Theorem 1, from those for $\underline{\underline{S}}$, thus reversing the procedure of the previous section. The key results are in the next two lemmas.

LEMMA 3. Let $\alpha, \beta$ be mops in $\underline{\underline{R}}$. Then $\alpha \underline{\underline{R}}^{\beta}$ if and only if $\alpha_{*} \lambda_{\underline{\underline{S}}} \beta_{*}$

Proof. Lemma 2, part ( $i$, gives immediately the "only if" part of the assertion.

Conversely, suppose that $\alpha_{*} \underline{\underline{\underline{S}}}^{\beta}{ }_{*}$. Let $\alpha$ denote $(X, \alpha, Y)$. Then $\beta$ denotes $(U, B, Y)$, for some set $U$, and there exists $(P(X), \xi, P(U))$, say, a map in $\underline{\underline{\mathrm{S}}}$, such that

$$
\left(P(X), \alpha_{*}, P(Y)\right)=(P(X), \xi, P(U))\left(P(U), \beta_{*}, P(Y)\right) .
$$

In particular, $\alpha_{*}=\xi \circ \beta_{*}$.

Denote by $\mu$ the largest subset of $X \times U$ such that $\mu \circ \beta \subseteq \alpha$. Thus

$$
\mu=\{(x, u) \mid(u, y) \in \beta \text { implies }(x, y) \in \alpha\} .
$$

We show that $\alpha \subseteq \mu \circ \beta$. 
Let $(x, y) \in \alpha$. Then

$$
y \in\{x\} \alpha_{*}=\{x\}\left(\xi \circ \beta_{*}\right) ;
$$

so, since $\beta_{*}$ maps the empty set onto the empty set, there exists $u$ in $U$ such that $y \in\{u\} \beta_{*}$ and $u \in\{x\} \xi$. Then $(x, u) \in \mu$; for, if $(u, z) \in \beta$, then $z \in(\{x\} \xi) \beta_{*}=\{x\}\left(\xi \circ \beta_{*}\right)=\{x\} \alpha_{*}$, that is $(x, z) \in \alpha$. Hence $(x, y) \in \mu \circ \beta$.

It follows that $\alpha=\mu \circ \beta$ and that $\alpha \lambda_{\underline{\underline{R}}} \beta$; which completes the proof of the lemma.

LEMMA 4. Let $\alpha, \beta$ be mops in $\underline{\underline{R}}$. Then $\alpha \underline{\underline{\rho}}^{\beta}$ if and only if $\alpha_{*} \rho_{\underline{\underline{S}}}^{\beta} *$

Proof. Lemma 2, part ( $i i)$, suffices to give the "only if" part of the as sertion.

Conversely, suppose that $\alpha_{*} \rho_{\underline{\underline{S}}} \beta_{*} \cdot$. Then if $\alpha$ denotes the map $(X, \alpha, Y), \beta$ must denote $(X, \beta, V)$, for some set $V$, and there must exist $(P(V), \eta, P(Y))$ such that $\alpha_{*}=\beta_{*} \circ \eta$.

Then

$$
\alpha_{*} \circ \alpha_{*}^{-1}=\beta_{*} \circ \eta \circ \eta^{-1} \circ \beta_{*}^{-1} \supseteqq \beta_{*} \circ{ }^{2} P(V) \circ \beta_{*}^{-1}=\beta_{*} \circ \beta_{*}^{-1} .
$$

Hence, in particular, for $A, B \in P(X)$,

$$
A B_{*} \subseteq B B_{*} \text { implies } A \alpha_{*} \subseteq B \alpha_{*} \text {; }
$$

for, if $A \beta_{*} \subseteq B \beta_{*}$, then $(A \cup B, B) \in \beta_{*} \circ \beta_{*}^{-1} \subseteq \alpha_{*} \circ \alpha_{*}^{-1}$, so that $A \alpha_{*} \subseteq B \alpha_{*}$.

Denote by $v$ the largest subset of $V \times Y$ such that $\beta \circ v \subseteq \alpha$. Thus

$$
\nu=\{(v, y) \mid(x, v) \in \beta \text { implies }(x, y) \in \alpha\} .
$$

We show that $\alpha \subseteq \beta \circ \nu$.

Let $(x, y) \in \alpha$. Then $\{x\} \beta_{*}$ is not empty. For, denoting the empty set by $\square$, if $\{x\} \beta_{*}=\square$, then $(\square,\{x\}) \in \beta_{*} \circ \beta_{*}^{-1} \subseteq \alpha_{*} \circ \alpha_{*}^{-1}$; and so $\{x\} \alpha_{*}=\square \alpha_{*}=\square$, conflicting with the assumption that $y \in\{x\} \alpha_{*}$. 
Set $\{x\} B_{*}=\left\{v_{i} \mid i \in I\right\}$. Suppose that $(x, y) \notin \beta \circ v$. This can happen only if $\left(v_{i}, y\right) \notin \nu$ for all $i$ in $I$ and this means that, for each $i$ in $I$, there exists $c_{i}$, say, in $X$, such that $\left(c_{i}, v_{i}\right) \in \beta$ and $\left(c_{i}, y\right) \notin \alpha$. Set

$$
C=\left\{c_{i} \mid i \in I\right\} \text {. }
$$

Then

$$
C B_{*} \supseteq\left\{v_{i} \mid i \in I\right\}=\{x\} B_{*},
$$

so that, by condition (1),

$$
C \alpha_{*} \supseteq\{x\} \alpha_{*}
$$

Thus, since $y \in\{x\} \alpha_{*}$, there exists $c_{i}$ in $C$ such that $y \in\left\{c_{i}\right\} \alpha_{*}$, that is $\left(c_{i}, y\right) \in \alpha$. This is a contradiction.

Hence we have $\alpha=\beta \circ v^{\prime}$, whence it follows that $\alpha \rho_{R} \beta$.

We can use the functor $\alpha+\alpha^{*}$ to obtain further useful characterizations of left and right divisibility. Observe that $\alpha \lambda_{\mathrm{R}}{ }^{\beta}$ if and only if $\alpha^{-1} \rho_{\underline{R}} \beta^{-1}$ and that $\alpha^{*}=\left(\alpha^{-1}\right)_{*}$. From these observations we easily infer from Lemmas 3 and 4 the following results.

LEMMA 5. Let $\alpha$ and $\beta$ be maps in $\underline{\underline{R}}$. Then $\alpha \lambda_{\underline{R}}{ }^{\beta}$ if and only if $\alpha^{*} \underline{\underline{S}}^{\beta^{*}}$.

LEMMA 6. Let $\alpha$ and $\beta$ be maps in $\underline{\underline{R}}$. Then $\alpha \underline{\underline{R}}^{\beta}$ if and only if $\alpha^{\star} \lambda_{\underline{\underline{S}}}^{\beta^{*}}$.

We now interpret our results and show that, for the category $\underline{R}$, we have obtained an alternative approach to Zareckil's characterization of left and right divisibility in $R$.

Let $(X, \alpha, Y)$ be a map in $\underline{\underline{R}}$. We set $M_{\alpha}=\nabla\left(\alpha_{*}\right)$ and $L_{\alpha}=\left\{X \backslash B \mid B \in \nabla\left(\alpha^{*}\right)\right\}$. Then $L_{\alpha}$ is a complete lattice of subsets of $X$ closed under arbitrary set-theoretic intersection and $M_{\alpha}$ is a complete 
lattice of subsets of $Y$ closed under arbitrary set-theoretic union. Moreover, the restriction $f_{\alpha}$, say, of $\alpha_{*}$ to $L_{\alpha}$, is a lattice isomorphism of $L_{\alpha}$ onto $M_{\alpha}[4, \S 1.7]$.

Conversely, given any complete lattice $L$, say, of subsets of $X$, closed under set-theoretic intersection, and a complete lattice $M$, say, of subsets of $Y$, closed under set-theoretic union, and an isomorphism $f$, say, of $L$ onto $M$, then there exists a unique binary relation $(X, \alpha, Y)$ such that $L_{\alpha}=L, M_{\alpha}=M$, and $f_{\alpha}=f \quad[4$, Theorem 1.8].

We shall call $\left(L_{\alpha}, M_{\alpha}\right)$ the pair of lattices of $\alpha$.

For any relations $\alpha$ and $\beta$,

$$
L_{\alpha}=\{X \backslash A \mid A \in M \underset{\alpha}{-1}\} \text {, }
$$

and

$$
L_{\alpha} \subseteq L_{\beta} \text { if and only if } M_{\alpha^{-1} \subseteq M_{\beta^{-1}}}
$$

Hence Theorem 1 and Lemmas 3, 4, 5 and 6 combine to give the next theorem.

THEOREM 3 (Zareckii). Let $\alpha, \beta$ be maps in $\underline{\underline{R}}$. Then

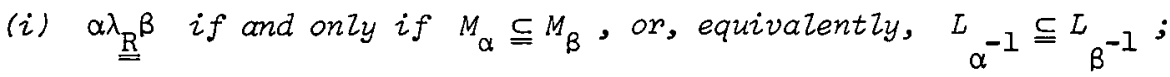
whence $\alpha \underline{\underline{R}}_{\underline{R}}^{\beta}$ if and only if $M_{\alpha}=M_{\beta}$ (or $\left.L_{\alpha^{-1}}=L_{\beta^{-1}}\right) ;$ and

(ii) $\alpha \rho_{\underline{\underline{R}}} \beta$ if and only if $L_{\alpha} \subseteq L_{\beta}$, or equivalently, $M_{\alpha^{-1}} \cong{ }_{\beta^{-I}}$; whence $\alpha \underline{\underline{R}}^{\beta}$ if and only if $L_{\alpha}=L_{\beta} \quad\left(\begin{array}{ccc}\text { or } & M_{\alpha^{-1}}=M_{\beta^{-1}}\end{array}\right)$.

As already remarked, because of Lemma 1 , Theorem 3 may be used to characterize $\lambda_{\underline{\underline{K}}}, L_{\underline{K}}, \rho_{\underline{K}}$ and $R_{\underline{K}}$ for $\underline{\underline{K}}$ any one of $\underline{\underline{S}}, \underline{\underline{P}}$ and $\underline{\underline{I}}$. We end with some comments upon these characterizations and their connexions with those of Theorem 1 . For brevity we restrict our remarks to $L_{\underline{\underline{K}}}$ and to $R_{\underline{\underline{K}}}$. 
If $\alpha$ is a map in $\underline{\underline{S}}$ then $M_{\alpha}$ is the lattice of all subsets of $\nabla(\alpha)$. Thus, if $\alpha, \beta$ are maps in $\underline{\underline{S}}$, from Theorem 3 we infer that $\alpha L_{\underline{\underline{S}}} \beta$ if and only if $P(\nabla(\alpha))=P(\nabla(B))$. But, for any sets $A$ and $B$, $P(A)=P(B)$ if and only if $A=B$. Hence $\alpha L_{\underline{S}} \beta$ if and only if $\nabla(\alpha)=\nabla(\beta)$, reproducing the condition of the Corollary to Theorem 1 . Again, from Theorem 3, $\alpha R_{\underline{\underline{S}}} \beta$ if and only if $M_{\alpha^{-1}}=M_{\beta^{-1}}$. It is readily verified that ${ }^{M} \alpha^{-1}$ is a complete atomic lattice with the equivalence classes of $\alpha \circ \alpha^{-1}$ as its atoms. A similar remark applies to $M_{\beta^{-1}}$. Hence $\alpha \underline{\underline{S}}_{\underline{S}} \beta$ if and only if $\alpha \circ \alpha^{-1}=\beta \circ \beta^{-1}$, reproducing again the condition of the Corollary to Theorem 1 .

For the category $\underline{\underline{P}}$ the interpretation is similar. Suppose that $\alpha$ is a map in $\underline{\underline{P}}$. Then $M_{\alpha}$ is again $P(\nabla(\alpha))$ and $M_{\alpha}{ }^{-1}$ is a complete atomic lattice with the elements of $\Delta(\alpha) /\left(\alpha \circ \alpha^{-1}\right)$ as its atoms. Thus, if $\alpha, \beta$ are maps in $\underline{\underline{P}}$, then $\alpha \underline{\underline{p}}_{\underline{p}}$ if and only if $\nabla(\alpha)=\nabla(\beta)$ and $\alpha \underline{\underline{p}}_{\underline{p}} \beta$ if and only if $\Delta(\alpha) /\left(\alpha \circ \alpha^{-1}\right)=\Delta(\beta) /\left(\beta \circ \beta^{-1}\right)$, that is if and only if $\alpha \circ \alpha^{-1}=\beta \circ \beta^{-1}$.

The interpretation for $\underline{\underline{I}}$ is now clear.

\section{References}

[1] W.D. Munn, "Fundamental inverse semigroups", Quart. J. Math. Oxford (2) 21 (1970), 157-170.

[2] G.B. Preston, "Embedding any semigroup in a D-simple semigroup", Trans. Amer. Math. Soc. 93 (1959), 351-355.

[3] N.R. Reilly, "Embedding inverse semigroups in bisimple inverse semigroups", Quart. J. Math. Oxford (2) 16 (1965), 183-187. 
[4] K.A. ZareckiY, "The semigroup of binary relations" (Russian), Mat. Sb. (N.S.) 61 (103) (1963), 291-305.

Monash University,

clayton,

victoria. 